\title{
E-learning: Issues and Challenges
}

\author{
Hemant Rana \\ SOCIS \\ IGNOU \\ New Delhi-68
}

\author{
Rajiv \\ SOCIS \\ IGNOU \\ New Delhi-68
}

\author{
Manohar Lal \\ SOCIS \\ IGNOU \\ New Delhi-68
}

\begin{abstract}
Providing education and training to the masses on gigantic scale, for economic survival and to meet the ever-changing requirements of the society and also to meet the individual's special requirements and tastes, is not possible through the conventional system of education based on brick-and-mortar schools, colleges and universities. The World Wide Web (WWW) is being used to improve communication, collaboration, sharing of resources, promoting active learning, and delivery of education in distance learning mode. Distance Education, E-learning and Virtual Universities may provide the desired solution. E-Learning may be taken as the latest form of distance learning mediated by state-of-art technologies like Internet and World-Wide-Web. In recent years, many of the universities and educational institutions worldwide offer online services such as for admissions, virtual (online) learning environments in order to facilitate the lifelong learning and to make this compatible with other educational management activities. Current e-learning research brings together pedagogical, technical and organisational concerns within a wider set of socio-cultural factors. Understanding issues \&challenges in respect of elearning is of significant importance to the research communities involved in e-learning and will have a significant role in forming future practices. In consulting the INDIA research community, a number of research issues \& challenges are required to be addressed to promote more efficient learning techniques.
\end{abstract}

\section{General Terms}

e-learning, education.

\section{Keywords}

distance education, e-learning, education, WWW.

\section{INTRODUCTION}

In the previous (i.e., 20th) century, we witnessed an unprecedented technological advancement - specially, in the fields of electronic computing and communications changing our lives and our perspective of the world in an unimaginable way. The computing and communications revolution, while improving quality of our life, has also brought in its wake a number of challenges. The world has become a (global) village [7]. The global village view of the world presents us with a number of formidable challenges including the ones due to the following facts:

- The patterns of trade, competitions and technological innovation are changing at an everincreasing rate.

- There is exponential growth of knowledge, again, at an ever-increasing rate.
- There are world-wide social concerns for freedom and general quality of life.

- Demand for universal access in respect of opportunities for relevant and quality education especially for economic survival and advantage.

In view of the above-mentioned challenges, for a community or nation to survive and lead, it is essential that at least, its working-age group is provided means for mass learning/education so that it is well-equipped with latest required skills for various economic and intellectual activities.

Providing education and training to the masses on gigantic scale, for economic survival and to meet the ever-changing requirements of the society and also to meet the individual's special requirements and tastes, is not possible through the conventional system of education based on brick-and-mortar schools, colleges and universities. Distance Education, Elearning and Virtual Universities may provide the desired solution.

Continuous efforts in the field of communication technologies have resulted in more efficient \& cost effective mode of learning as compared to traditional strategies of learning. Now students \& educators have better control on the process of teaching \& learning. However, from academic point of view, two questions may arise immediately:

What does e-Learning mean exactly?

Is e-Learning really the best way to acquire knowledge or education?

The answer to the first question cannot be given exactly, because it is still a topic of research and discussions are still going on. The concept like e-Learning is relatively a new term and has been evolving over number of previous years.

Through this paper, author is trying to focus on various issues and challenges in implementing E-learning system. Elearning is a very broad area of research in terms of education system. In this paper, it starts with an introduction on elearning system and followed by the some definitions on elearning and distance education. Section 3 is all about the discussion on issues and challenges in different areas of elearning domain. In section 4, introduces analysis and recommendations to overcome these challenges. And finally section 5 tells you about future scope of e-learning.

\section{SOME RELEVANT DEFINATIONS \& EXPLANATIONS}

In order to set the subject matter in proper perspective it begin with some brief descriptions [8].

Distance Education: As, later on, they are going to mention that e-learning is the state-of-art computing and communication mediated Distance Education; it is desirable 
to enumerate the characteristic features of Distance Education, which are identified by Keegan (1980) [1] , one of the pioneers in the discipline, as follows:

- The separation of learner and teacher

- Influence of an educational organization

- The use of media as a medium between a teacher and a learner(like, TV, RADIO etc )

- Facilitate two-way exchange of information

- Learners as an individual rather than in group; and

- Educators as an industrialized form

E-Learning: Rosenberg (2001) [2] Defines the term elearning as, the use of Internet technologies to deliver a broad array of solutions that enhance knowledge and performance". e-learning is based on three fundamental criteria suggested by Rosenberg [2]:

- Networked for instant updating, distribution, storage/retrieval and sharing of information.

- Content delivery via computer using WWW.

- It focuses on the broadest view of learning and learning solutions.

Thus, e-Learning may be taken as the latest form of distance learning mediated by state-of-art technologies like Internet and World-Wide-Web. They must remember that e-Learning is much more than online training or Computer-Based Training (CBT), encompassing knowledge management and electronic performance support, Computer -conferencing enabling group communication, enabled by Internet and WWW is one the key characteristics of e-learning which makes it qualitatively much superior to the conventional distance learning paradigm [2], [4] , [5].

Longmire (2001) states "e-Learning covers a wide set of applications and processes such as computer-based learning systems, Web-based learning systems, virtual classrooms, and digital collaborative learning GroupWare packages. ELearning content is mainly delivered via Internet, intranet/extranet, audiotape and videotape; satellite broadcast, interactive TV, DVD and CD-ROM, and the still to emerge wireless application protocols (WAP) "[3 ].

Computer - based conferencing that enables group communication, and is itself enabled by Internet and WWW is one of the key characteristic of e-Learning which makes it qualitatively much superior to the conventional distance learning paradigm.

Key attributes of e-Learning, as subsumed by the above definition, may be explicitly stated as:

- Many-to-many (i.e. group) communication

- Any place (place independence)

- Any time (asynchronicity, time independence)

- Text, enhanced by multi-media and

- Computer-conferencing (i.e., computer mediated messaging)

In order to facilitate learning \& education, web is being used as the backbone and we cannot underestimate its value in delivery and promoting effective learning to the people.

For about last two decades, the World Wide Web(WWW) is being used to improve communication, collaboration, sharing of resources, promoting active learning, and delivery of education in distance learning mode[6][16]. The WWW helps teachers in planning suitable online delivery structure, sharing goals of learning, and activities for their courses.

In recent years, many of the universities and educational institutions worldwide offer online services such as for admissions, virtual (online) learning environments in order to facilitate the lifelong learning and to make this compatible with other educational management activities[9]. For example, a teacher may create a purely Web-based delivery system including online handouts in respect of student's activities, projects and lists of resources for reference. The students and other learners may access web-based material anytime from anywhere in the world, being connected through Internet[15].

\section{ISSUES \& CHALLENGES IN E- LEARNING}

\subsection{Technological Challenges}

The e-learning raises significant challenges in the technological research area. For development of e-learning resources that meet the users requirement need to be addressed. The technological challenges of e-learning can be considered as a two key technological research areas.

\subsection{Development of New Forms of Learning community and Interactive Learning}

In e-learning environments interaction, cooperation and community play an important role to support learning. The developments in the area of e-learning environments provide new forms of interaction for learning experience. It generates new relationships between learner and computer and also form a new learning community. Key issues include:

- New forms of multimodal interface to support learning.

- New techniques to understand and support learning communities.

- The development of systems to support mobile communities of learners.

- Personalisation techniques that meet user personal needs and current activity.

- Techniques to promote and support interaction.

- Discovery of new learning communities.

- Support for time to time assessment services.

\subsection{Developing New Knowledge Facilities for e-learning}

e-learning environment needs to support the rapid increase in the size and variety of data by appropriate semantic services. The semantic services generate a surrounding semantic context for learning support. Research that needs to work on:

- Development of learning and reasoning theories for uncertain and incomplete knowledge.

- $\quad$ Support for the development of large-scale learning facilities.

- Support for a dynamic learning process.

- Support for information sharing across different learning facilities.

- Developments of lightweight knowledge capture technique for promotion of lifelong learning. 
- Development of learning support services as per the requirement of different domains and users.

\subsection{Research Issues for e-learning}

Current e-learning research brings together pedagogical, technical and organisational concerns within a wider set of socio-cultural factors. These factors influence the research agenda in e-learning system. Understanding these broader social and cultural issues is of significant importance to the research communities involved in e-learning and will have a significant role in informing future practices. In consulting the INDIA research community, a number of research issues emerged:

Interdisciplinary, and the notion of multiple voices, is a defining characteristic of the area. How do different research perspectives influence the overall area? How do problems in the practice of different disciplines differ in the adoption and use of learning technologies?

Access and inclusion, includes issues around the widening participation agenda. What are the barriers to inclusion and what are the issues surrounding the extent of the digital divide?

Change, and its relationship to learning technologies. How does change impact upon motivational issues? What are the drivers and rationales for change? What are appropriate strategies for managing changes and mechanisms for their implementation?

Convergence and interoperability, in terms of exploration of different forms of convergence (organisational, technological, pedagogical, sectoral, institutional, etc). How well do they understand issues of globalisation and scalability standards to support interoperability? These are complex relationships. A current focus is also on standardisation across the pedagogical, technical, organisational and human aspects versus critique of convergence.

Interactivity and social interaction: How does the interactivity of different tools relate to, or impact upon, the nature of the medium? How is interactivity expressed at different levels of organisations, and how have organisational boundaries and functional groupings blurred as a consequence of new technologies? What potential do the new technologies have to enhance communication and collaboration and also creating new communities and networks?

\section{ANALYSIS \& RECOMMENDATIONS}

According to the study, learning contents and learning communities got the highest priority in e-learning research. In these areas, lots of development has to be made. As technology is growing day by day, learner interest in elearning increases very rapidly. Now researcher has begun to work on the designing new e-learning methodologies that can work according to learners' interest and preference. For predicting learner interest, they have to study learner behavior, learning style by their online activities and search criteria.

Experts in the field believe that some of the most promising features of modern e-learning platforms will be Web with intelligence, i.e., an intelligent web. Applications will work intelligently with the efficient use of Human-Computer interaction (HCI) and intelligence. Different Artificial Intelligence (AI) based tools \& techniques (such as, rough sets, fuzzy sets, neural networks, machine learning etc) can be integrated with the e-learning applications to support intelligence [10].

In support of e-learning on the web, a new version of World Wide Web called Web 3.0 has been proposed as a possible future consisting of the integration of high-powered graphics (Scalable Vector Graphics or SVG) and semantic data. There have also been discussions around 3-D social networking systems and immersive 3-D internet environments that will take the best of virtual worlds (such as Second Life) and gaming environments and merge them with the Web. Web 3.0 based e-learning services will be having constructive impact on education. Web 3.0 technologies offer benefits of 3Dwikis, 3D Labs; Intelligent Agent based search engines, Virtual environments like Avatar, Semantic Digital Libraries that may result in added advantage in delivery of effective elearning to the mass [9][13].

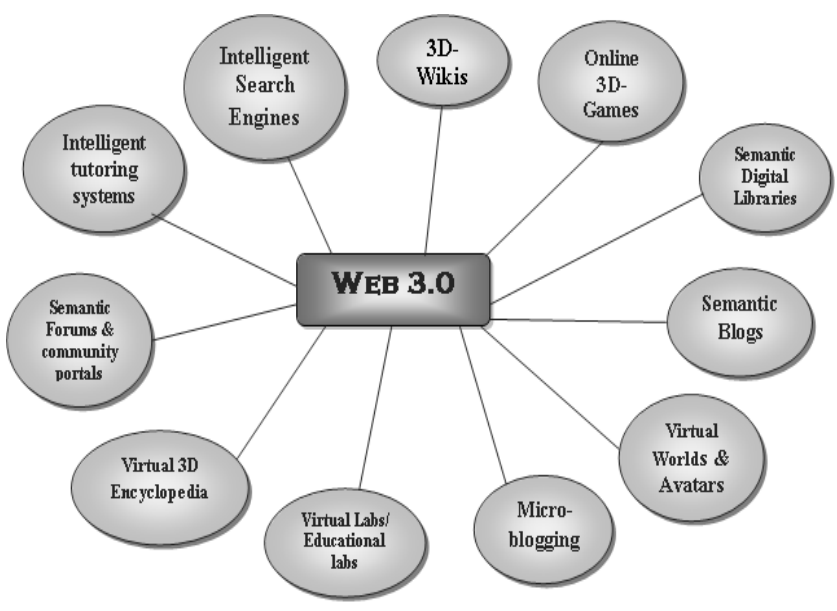

Fig 1: Web 3.0 Tools \& Services

Semantic web is another promising technology for realisation of e-learning requirement. In the simplest terms, we can define Semantic Web as a relationship between things, described in a manner which makes people and machines able to understand [9]. One of the objectives of Semantic Web is to identify, recognise and extract the exact required data that matches the keywords provided by the user. semantic web facilitates flexible and personalised access to the learning material. semantic web is suitable platform for implementation of e-learning environment because it provides ontology-based annotation of learning materials, ontology development and proactive delivery of the learning materials through e-learning systems [11].

e-learning is a complex system with multiple forms and different level of interest of learner. To make e-learning system to more interactive for learner, researchers need to take decision at every level of learning cycle. A Decision Support System (DSS) is an interactive information system that provides models, data manipulation tools and information. DSS helps to make decisions in semi-structured and unstructured situation [12]. By offering such approach, education system could play much better for student centric operation towards positive improvement of his performance [9]. 


\section{FUTURE OF E-LEARNING}

Over the past few years, adoption of technology in the education has increased significantly. Most of the universities like to invest in modern forms of learning, therefore it may be seen that the growth of e-learning is expanding tremendously. The new form of education through technology is attracting entrepreneurs around the world to invest and develop new forms of e-learning products.

According to recent studies done by Docebo[14] in 2014, the global market growth for Self-Paced E-Learning reached $\$ 35.6$ billion in 2011. The five- year compound annual growth rate for e-learning is estimated approximately $7.9 \%$. Hence, the global revenues should reach approximately $\$ 51.5$ billion dollars by 2016 as shown in figure 2 .

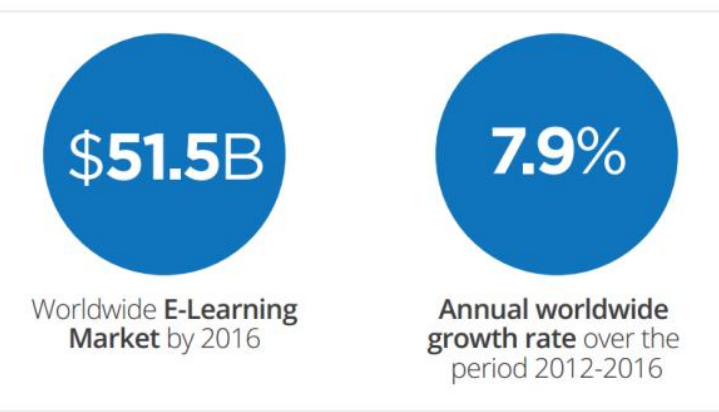

Fig 2: Global E-Learning Market [14]

Studies shows aggregate e-learning growth $7.9 \%$ annually worldwide but regional growth is somehow much more in some specific continents. According to figure 3, the highest growth rate is in Asia at $17.3 \%$, followed by Eastern Europe, Africa, and Latin America at 16.9\%, 15.2\%, and 14.6\%, respectively. Entrepreneurs from the world are attracting to this market as the revenues generated in this market are extremely high.

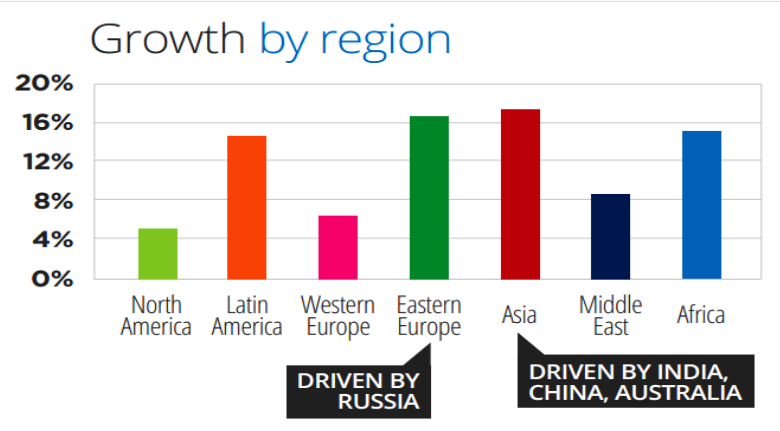

Fig 3: Regional E-Learning Market [14]

In Asia, specifically, Indian universities are focusing into effective higher distance education to provide cheaper effective education to the learners. India has become a mature player for higher distance education in the Asian E-Learning market.

\section{CONCLUSION}

This paper discusses about the formulation of the problem and their need and prospects in today's education field. We discussed why e-Learning is required at all, followed by relevant definitions quoting some pioneers in the field. Through this paper, we trying to focus on various issues and challenges in implementing E-learning system. E-learning is a very broad area of research in terms of education system. Web 3.0 technologies offer benefits of 3D-wikis, 3D Labs; Intelligent Agent based search engines, Virtual environments like Avatar, Semantic Digital Libraries that may result in added advantage in delivery of effective e-learning to the mass. Semantic web is another promising technology for realisation of e-learning requirement. In view of the facts that AI technologies plays significant role in almost every domain of human experience, specially, when the domain is complex. Modern e-learning platforms are required to be equipped intelligence, i.e., an intelligent web. Applications can work intelligently with the efficient use of Human-Computer interaction (HCI) and intelligence. Different Artificial Intelligence (AI) based tools \& techniques (such as, rough sets, fuzzy sets, neural networks, machine learning etc) can be integrated with the e-learning applications to support intelligence. . To make e-learning system to more interactive for learner, researchers need to take decisions at every level of learning cycle. A Decision Support System (DSS) is an interactive information system that provides models, data manipulation tools and information. DSS helps to make decisions in semi-structured and unstructured situation. By offering such approach, education system could play much better for student centric operation towards positive improvement of his performance.

\section{REFERENCES}

[1] Keegan, D. (1980). On Defining Distance Education: Distance Education 1(1) 13-36.

[2] Rosenberg, M. J. (2001). E-learning: Strategies for delivering knowledge in the digital age. New York: McGraw-Hill.

[3] Longmire, W. (2001). A Primer on Learning Objects. Managing Web-Based Training : ASTD.

[4] Tomei, A., (2003). Challenges of teaching with technology across the curriculum: issues and solutions. Information Science Publishing.

[5] Mortimore, P., (1999). Understanding pedology and its impact on learning. Paul Chapman Publishing Ltd.

[6] Rana, Hemant, Rajiv, and Prof. Manohar Lal. "Rough set based system for effective E-learning." Computing for Sustainable Global Development (INDIACom), 2014 International Conference on. IEEE, 2014; ISSN 0973-7529 and ISBN 978-93-80544-10-6.

[7] Harasini L., et al. (2001). The Virtual University: A State of the Art. Advances in Computers, Vol. 55, PP 147.

[8] Witten I.H., et al. (2000). Greenstone: A comprehensive Open-Source Digital Library Software System. ACM 2000 Digital Library, PP 113-121.

[9] Rajiv \& Manohar Lal, (2011). Web 3.0 in Education \& Research, BVICAM's International Journal of Information Technology (BIJIT), Vol. 3 No. 2; ISSN $0973-5658$.

[10] Robin D. Morris, (2011). Web 3.0: Implications for Online Learning, TechTrends Vol. 55, No. 1 Springer. 
[11] L. Stojanovic, S. Staab, and R. Studer, (2001). Elearning based on semantic web, WEBNET 2001.

[12] M.S. Velmurugan and K. Narayansamy, (2008). Application of decision support system in e-commerce, Communications of the IBIMA, Vol. 5.

[13] Han Xiaoting, Niu Li, (2010). Subject Information Integration of Higher Education Institutions in the Context of Web3.0, 2nd International Conference onIndustrial Mechatronics and Automation, 978-1-42447656-5/10, 2010, IEEE.

[14] E-Learning Market Trends \& Forecast 2014 - 2016 Report , A report by Docebo, March 2014, URL: http://www.docebo.com/landing/contactform/elearning- market-trends-and-forecast-2014-2016-doceboreport.pdf (accessed 30/06/14).

[15] Hemant Rana, Rajiv, Prof. Manohar Lal, (2014). Role of Artificial Intelligence based Technologies in e-learning, International Journal of Latest Trends in Engineering, Science and Technology (IJLTEST), Volume 1, Issue 5, May 2014, ISSN: 2055-530X(online), ISSN: 20555296(print), London, United Kingdom.

[16] Rana, Hemant, Rajiv, and Prof. Manohar Lal. "Rough set based system for effective E-learning." IEEE, 2014; ISBN: 978-93-80544-10-6. 